\title{
Research on Separation Variable Method in Mathematical Physics Equation
}

\author{
Xishan Yu \\ Applied Technology College of Dalian Ocean University, Dalian, Liaoning, China
}

\author{
Keywords: Separation Variable Method, Mathematical Physics Equation
}

\begin{abstract}
The separation variable method is one of the general methods to solve the boundary value problem of various types of linear partial differential equations. On the one hand, the vibration equation of the first kind of boundary condition is solved by using the separation variable method. On the other hand, the vibration equation of the second kind of boundary condition is solved by the method. The solution of the separation variable method is deepened by solving the equation, the basic idea of this method is to convert the partial differential equation satisfying the multivariate function into the ordinary differential equation of several unary functions, and obtain the solution of the corresponding equation by means of the existing mathematical knowledge.
\end{abstract}

\section{Introduction}

The great mathematician and philosopher Descartes once said, "All problems can be translated into mathematical problems, and all mathematical problems can be transformed into algebraic problems. All algebraic problems can be transformed into equations." As a communication class, electrons the core of the "mathematical physics equation" is to convert a physical problem into a mathematical problem and solve the problem of solving the problem (mainly the second order linear partial differential equation). Therefore, the main tool of the linear partial differential equation, the importance of the separation variable method is self-evident, so the "separation variable method" chapter is "mathematical physics equation" teaching focus and difficulty, and its system of teaching and learning also seem vital.

Mathematical physics equation is a discipline that studies the partial differential equations generated by physics and other natural sciences and technical sciences. It is a basic theory course with a wide range of applied background, whether it is engaged in basic research or engineering development. The complexity of the objective world leads to the complexity of describing the mathematical equations of the relationship, so that these partial differential equations contain more independent variables, the solution is quite complex. How to simplify the solution method becomes an important aspect of solving mathematical equations. The separation variable method is a common and important method for solving partial differential equations. The method can separate the partial differential equations into ordinary differential equations so that some partial differential equations become solvable. The solution of the mathematical solution of mathematical physics is only applicable to a very small number of certain solutions, and the separation variable method is a basic solution of the solution problem, which is applied to the initial boundary value problem on a large number of finite fields.

\section{The Related Research of Mathematical Physics Equation}

Mathematical physics equation is based on the partial differential equation with physical background as the main object of the study. By teaching the derivation, initial condition and boundary condition of three typical equations, the correct formulation of the partial differential equation and the solution so that students master the most basic theory of partial differential equations, methods and techniques to understand the use of mathematical methods to solve practical problems in the general process, the formation of a certain degree of rational thinking and a high degree of analysis of the problem, the ability to solve the problem. As the engineering mathematics course after the university 
mathematics physics course such as "higher mathematics", "linear algebra", "university physics", this course is the professional foundation of undergraduate students of electronic information, communication, computer science, physics, mechanics and so on course. The purpose of the course is to provide the necessary mathematical foundation and tools for the study of subsequent professional courses, to consolidate and deepen the mathematical knowledge learned in the math curriculum, and to provide initial training for students to apply mathematical tools to solve practical problems. From the perspective of the role of university mathematics education, from the curriculum structure and characteristics of view, mathematical physics equation is the most able to reflect the characteristics of mathematics quality education courses. It aims to cultivate students' rational thinking, application analysis ability and innovation consciousness, and strive to improve the quality of mathematics as the primary task of teaching and learning. Through years of teaching practice, we feel that in the continuous adjustment of the number of hours of teaching conditions, to complete the established teaching tasks, must be from the teaching content, teaching methods, assessment methods, auxiliary means and other aspects of reform.

Most of the problems studied by mathematical equations are derived from the practical problems in physics and mechanics, and have extensive links with various fields of natural sciences. The mathematical methods used to deal with these practical problems are inextricably linked to the maths courses that students have learned. Therefore, the "Mathematical Equality" course is an important bridge between communication mathematics and practical problems of natural science and engineering technology. On the one hand, this course can be used to master the basic courses of mathematics, and on the other hand, learn how to use mathematical theory to solve practical problems. Of course, as a combination of mathematical theory and practical problems of the course, the course involves complex content, many students learn this course also feel a certain degree of difficulty. In addition to introducing the basic content of the course, teachers should highlight their basic mathematical ideas, and strive to make students learn the knowledge at the same time, focusing on understanding the mathematical ideas. And the influence of mathematics thought on the students and the formation and improvement of students' mathematics accomplishment are especially important. The most basic mathematical thought in the "mathematical equation" is the idea of "transformation" in mathematics, that is, in the study and answer the mathematical problems, the complex or new problems through the appropriate transformation into a simple or already studied the problem, and ultimately solve the problem. The idea of "transformation" in the "mathematical equation" reflects the fact that people are constantly simplifying and making it difficult to understand and understand the process of knowledge, which in fact reflects the general law of scientific research and the basic method. In the classroom teaching very attention to highlight this mathematical thinking, not only to enable students to master the necessary mathematical knowledge, but also enable students to learn scientific thinking.

In addition, the concept should pay attention to clarify the actual background, so that students understand the practical problems as the background to establish the mathematical model of the basic methods and specific steps to understand the so-called mathematical physics equation is to use mathematical language to describe and express the physical phenomena and practical problems, from The beginning of the establishment of the mathematical model, to the establishment of the basic concepts and the main results of the elaboration of the whole process, must grasp and explain the physical background associated with it. This will make the mathematical concepts and results have a visual, both easy to understand and memory students, but also help to develop students' mathematical imagination. The focus of teaching should be on the introduction of basic concepts, basic methods, basic ideas, highlighting the ability to calculate, the application of training. In the teaching of the concept, as far as possible without strict "definition" in the form, try to dilute the strict theoretical proof to reduce the mathematical form of the sense of abstraction, as far as possible close to the student's actual description and vivid analogy, dilute the theory, the academic form of mathematics into students easy to accept the form of education. In the introduction of the basic theorem, do not rigidly adhere to the "theorem --- proof" of a single model, some only give the conclusion that the theoretical reduction, highlighting the application of the conclusion, that is, "instrumental". For the 
relatively strong abstract content, focus on the introduction of typical examples of examples, and through examples to gradually expand the theory to guide students to think about the conclusions. For example, in teaching the Poisson formula of the three-dimensional wave equation, the whole mathematical derivation makes the engineering students sound very troublesome. In teaching, we combine the physical meaning of the Poisson formula, try to simplify the proof, and give a detailed example Method of Solving Formula.

\section{The Overview of the Separation Variables Method}

The key to the separation variable method is to replace the probabilistic solution of the variable into the partial differential equation, which is decomposed into several ordinary differential equations. The problem is transformed into the ordinary differential equation. On the other hand, the condition of homogeneous boundary condition is transformed into the additional condition of ordinary differential equation. These conditions constitute the problem with the corresponding ordinary differential equation. Although we are from the standing wave leads to solving the problem of clues, in fact, the whole process with the standing wave and no special relationship, from the mathematical point of view can be applied to linear homogeneous equations and linear homogeneous boundary conditions of a variety of solutions problem. This method, according to its characteristics, is called the separation variable method.

The separation variable method, that is, for the partial differential equation, can be expressed in the form of a variable separation state. Assuming that the solution problem has the solution of the form of the variable separation, the solution is substituted into the partial eccentric equation, and the ordinary differential equation with the invariant constant can be obtained. When these special values are taken, some equations can satisfy the homogeneous condition Non-zero solution. For the separation variable method, it can be carried out in the Cartesian coordinate system, the spherical coordinate system, the cylindrical coordinate system and so on. With the different coordinate system, some definite differential equations can be found in the solution of the problem, with different solutions, functions can be functions such as Bessel functions. Since the theoretical basis of the separation variable method is linear superposition principle, it can only solve the linear solution problem. When dealing with the problem of nonhomogeneous generalized equations, it can be regarded as the result of several types of superposition, break down". Although there is a wide range of applications in the solution of mathematical equations, there are many limitations, which are limited by the principle of linear superposition and can only solve the linear problem of homogeneous boundary conditions. It can also be used, but with other methods), and secondly, the separation variable method is too harsh on the boundary conditions, and the solution of the separation variable method is generally an infinite series, which is an approximate solution in the error range.

\section{The Separation Variable Method to Solve the Mathematical Physics Equation}

The proposed method of separation variable is inspired by the problem of "standing wave". "Standing wave" is a common form of vibration phenomenon. The partial differential equation describing the "standing wave" can be expressed in the form of a variable separation state. Although we are from the standing wave leads to solving the clue, in fact, the whole process with the standing wave and no special relationship. In short, the separation variable method is to use the linear and homogeneous properties of the equation and boundary conditions. Firstly, the partial differential equation is separated into the ordinary differential equation, and the special solution satisfying the equation and the boundary condition is found. Then, make it meet the initial conditions, the equation is solved. Separation variable method is an important application method in mathematical physics equation, and it has a very important application in solving common partial differential equations. In the field of physics, there are many problems can be attributed to partial differential equations, therefore, the separation variable method in the physical application is also very wide. In this paper, we give some special cases and hope that we can establish the organic connection between 
mathematics and physics through the study of the separation variable method so as to make the mathematical method better serve the physical problem

\section{The Application of the Separation Variable Method in Solving Mathematical Physics Equation}

Find the initial boundary value problem for homogeneous equations with homogeneous boundary conditions (for example) to study the free vibration of uniformly uniform strings at both ends.

It needs to solve the initial boundary value problem of the nonhomogeneous equation with homogeneous boundary condition by separating the variable method. Firstly, the initial boundary value problem is decomposed into two initial boundary values according to the superposition principle and one is the non-homogeneous initial The Initial Boundary Value Problem of Homogeneous Equation. And the other is the initial boundary value problem of the nonhomogeneous equation with homogeneous initial conditions. The initial boundary value problem can be transformed into the homogeneous equation with nonhomogeneous initial conditions.

It needs to solve the initial boundary value problem with nonhomogeneous boundary conditions. If the boundary conditions are nonhomogeneous, the boundary conditions are first homogeneous. Is to choose a function with the unknown u has the same boundary conditions known function U, under normal circumstances we take the most simple linear function. And then transform $\mathrm{V}=\mathrm{u}-\mathrm{U}$, bring about the initial boundary value problem on u, and derive a new initial boundary value problem for $\mathrm{V}$, which is the boundary condition that V satisfies.

\section{Conclusions}

The separation variable method is to convert the partial differential equation into an ordinary differential equation. First of all, according to the initial boundary value to see whether the need for boundary conditions homogeneous, and then see whether the need for homogeneous system. The most important part is to solve the eigenvalue problem. Whether the method is effective, mainly depends on whether the eigenvalue exists, whether the feature function family is orthogonal, and whether the given function can be expanded by the feature function family. If the case is a triangular function family, we can solve the Fourier series.

\section{References}

[1] B. N. Ma, Mathematical Physics. M. Hefei: China University of Science and Technology Press. 2010 146-147.

[2] H.X.Zeng, Electromagnetic Field and Electromagnetic Wave. M. Beijing: Higher Education Press. 2005 55-57.

[3] J.H.Xue, On the Separating Variable Method J. Cangzhou Teachers College, 32(2012) 189-190.

[4] Y.J.Qi, Differential Equations and Case Analysis of Differential Equations J. Technology Horizon 4(2007) 38-40. 